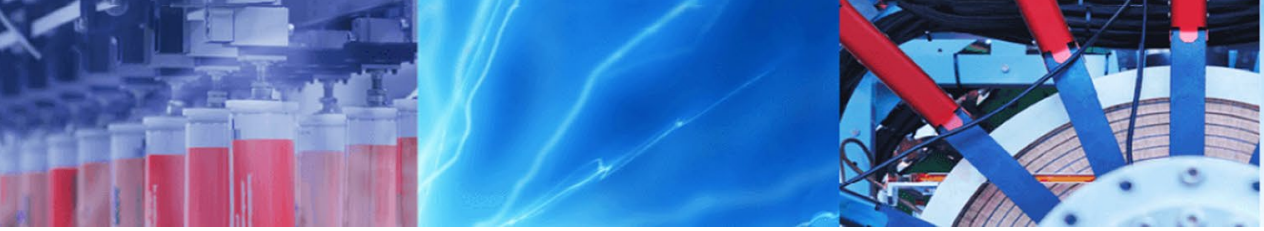

Research Article

\title{
Analytical equations based prediction approach for PM2.5 using artificial neural network
}

\author{
Jalpa Shah ${ }^{1}$ (D) Biswajit Mishra ${ }^{2}$
}

Received: 31 January 2020 / Accepted: 4 August 2020 / Published online: 14 August 2020

(c) Springer Nature Switzerland AG 2020

\begin{abstract}
The worldwide, particulate matter pollution is considered one of the deadliest types of air pollution due to its significant impact on the global environment and human health. The particulate matter (PM2.5) plays a key role in evaluating the air quality index. However, the conventional PM2.5 monitoring instruments used by the air quality monitoring stations are costly, bulkier, time-consuming, and power-hungry. Furthermore, due to limited data availability and non-scalability, it is challenging to provide high spatial and temporal resolution in real-time. To overcome these challenges, we present analytical equations based prediction approach for PM2.5 using an artificial neural network. Moreover, we contribute the correlation study between PM2.5 and other pollutants using a large authenticate data set of Central Pollution Control Board online station, India. The correlation study reveals the strong correlation of eight pollutants with PM2.5, which found useful for the proposed prediction model and future research work. The computation of the proposed analytical equation using a low-cost processing tool (excel sheet) demonstrates a good match between predicted and actual results. Additionally, the derived analytical equation for the prediction can be computed using a wireless sensor node which ultimately eliminates the need for costly propriety tools. The performance of proposed analytical equation for prediction show root mean square error and coefficient of determination $\left(R^{2}\right) 1.80 \mu \mathrm{g} / \mathrm{m}^{3}$ and 0.99 respectively using eight correlated predictors. The recalibrated prediction model with three correlated predictors show RMSE of $7.54 \mu \mathrm{g} / \mathrm{m}^{3}$ and $R^{2}$ of 0.97 and proves the effectiveness of the proposed approach in implementation using minimum power-hungry gas sensors on the WSN. Therefore, obtained results demonstrate that the proposed approach is one of the promising approaches for monitoring PM2.5 without power-hungry gas sensors and bulkier analyzers.
\end{abstract}

Keywords Prediction model · PM2.5 · Correlation · Artificial neural network · Air pollution monitoring · Machine learning

\section{Introduction}

Maintaining air quality is a prominent global challenge due to the adverse effects of air pollution on human health and the environment. Worldwide rapid depletion of forests, increased industrialization, and transportation is the major reason for air pollution. In India, the Central Pollution Control Board (CPCB) has established National Air Quality Monitoring Programme (NAMP) [1] to determine the status and trends of ambient air quality and executing correcting actions for the same. The NAMP gathers environment quality information by monitoring meteorological parameters such as temperature, humidity, atmospheric pressure, wind speed and pollutants such as carbon dioxide $\left(\mathrm{CO}_{2}\right)$, carbon monoxide $(\mathrm{CO})$, nitrogen dioxide $\left(\mathrm{NO}_{2}\right)$, sulphur dioxide $\left(\mathrm{SO}_{2}\right)$, volatile organic compounds (VOCs), suspended particulate matter (SPM), and ozone $\left(\mathrm{O}_{3}\right)$. Furthermore, monitored pollutants data is converted

\footnotetext{
$\bowtie$ Jalpa Shah, jalpa.iiscian@gmail.com; Biswajit Mishra, biswajit_mishra@daiict.ac.in | ${ }^{1}$ Electronics and Communication Engineering Department, Amrita School of Engineering, Bengaluru 560035, India. ${ }^{2}$ Dhirubhai Ambani Institute of Information and Communication Technology, Gandhinagar 382007, India.
} 
by NAMP in to air quality index (AQI) [2] at approximately 683 monitoring stations covering 300 cities/towns of 29 states and 6 union territories of the country. The AQI [3] provides information about air quality and health risks associated with it in the form of color-coded classifications, which is easy for the public to understand. The computation of AQI needs minimum three pollutants out of which one should be the Particulate Matter 2.5 (PM2.5) which is very harmful to human health.

Besides gaseous pollutants, the atmosphere can also be polluted by particles. Particle pollution is a mixture of solids and liquid droplets floating in the air. The particles are classified as: coarse, fine, and ultrafine. Coarse particles have a diameter of between 2.5 and $10 \mu \mathrm{m}$ and quickly get down by gravity or are washed out by rain. Sources for this kind of particles are crushing or grinding operations and dust stirred up by vehicles on roads. PM10 is particulate matter $10 \mu \mathrm{m}$ or less in diameter. Whereas fine particles $(0.1-2.5 \mu \mathrm{m}$ in diameter) and ultrafine $(<0.1 \mu \mathrm{m}$ in diameter) particles remain in the atmosphere for a longer time and affects human health. Particles less than $2.5 \mu \mathrm{m}$ in diameter are referred as fine particles. PM2.5 are fine particles of $2.5 \mu \mathrm{m}$ in diameter or smaller and can only be seen through an electron microscope. PM2.5 poses a major concern for human health as due to its small size $(<2.5 \mu \mathrm{m})$ they can directly enter into the lungs [4]. PM2.5 comes either from primary sources or from secondary sources. The primary sources can be vehicles, power plants, wood burning, industrial processes, forest or grass fires, and agricultural burning processes. The secondary sources are precursor emissions such as sulfur dioxide $\left(\mathrm{SO}_{2}\right)$, oxides, volatile organic compounds (VOCs), and ammonia $\left(\mathrm{NH}_{3}\right)[4,5]$.

The classification of both direct and indirect measurements for outdoor environmental monitoring is shown in Fig. 1. In direct methods, dedicated instruments such as analyzers [45], aethalometer [6], samplers [7-9] and in certain cases wireless sensor nodes (WSNs) [10-13] are used. Aethalometer and analyzers provide the pollutants'values directly but lack portability and are often expensive. In air pollution monitoring using samplers, offline analysis is done in specialized laboratories. Whereas in wireless sensor nodes (WSNs), the measurement of pollutants is done using on-board gas sensors and is often a cost-effective solution compared to other monitoring methods [14]. An example of such a system deployed in a New York subway is discussed in [6].

The indirect measurements [15-31, 33-39] used prediction approach based on the past data of the predictand, pollutants and/or meteorological parameters. These pollutants and meteorological parameters are correlated with the predictand [32, 40-42]. The PM2.5 (predictand) can be forecasted based on the data of pollutants and meteorological parameters which are correlated with PM2.5. The performance comparison of different prediction techniques of greenhouse gas is discussed in [48]. In [15], a comparison of different topologies of a neural network is presented for a prediction model of PM2.5. A neural network-based prediction model for PM10 using previous days data for PM10, cloud cover, boundary layer height, wind direction and day of the week is discussed in [16]. For the prediction of PM2.5 and $\mathrm{O}_{3}$, the empirical nonlinear regression model was designed [17] using meteorological parameters and past PM2.5 data. In [18] feed-forward neural network is used for prediction of PM2.5 based on past values of PM10, PM2.5 and some observed and forecasted meteorological parameters. In $[19,20]$, prediction based on ANN using past data of $\mathrm{O}_{3}$ is discussed. A multilayer perceptron (MLP) neural networkbased prediction model [21] for $\mathrm{NO}$ and $\mathrm{NO}_{2}$ pollutants is developed using past data of pollutants. In [22] prediction model show MLP neural network has better performance than multiple linear regression (MLR) model. Results in [23] demonstrates the better performance of ANN compared to MLR for prediction of PM2.5 in the agricultural park. Comparison results of MLR and ANN in [24], for prediction of PM2.5 represents the better performance of ANN. Feed
Fig. 1 Direct and indirect methods for outdoor environment quality monitoring

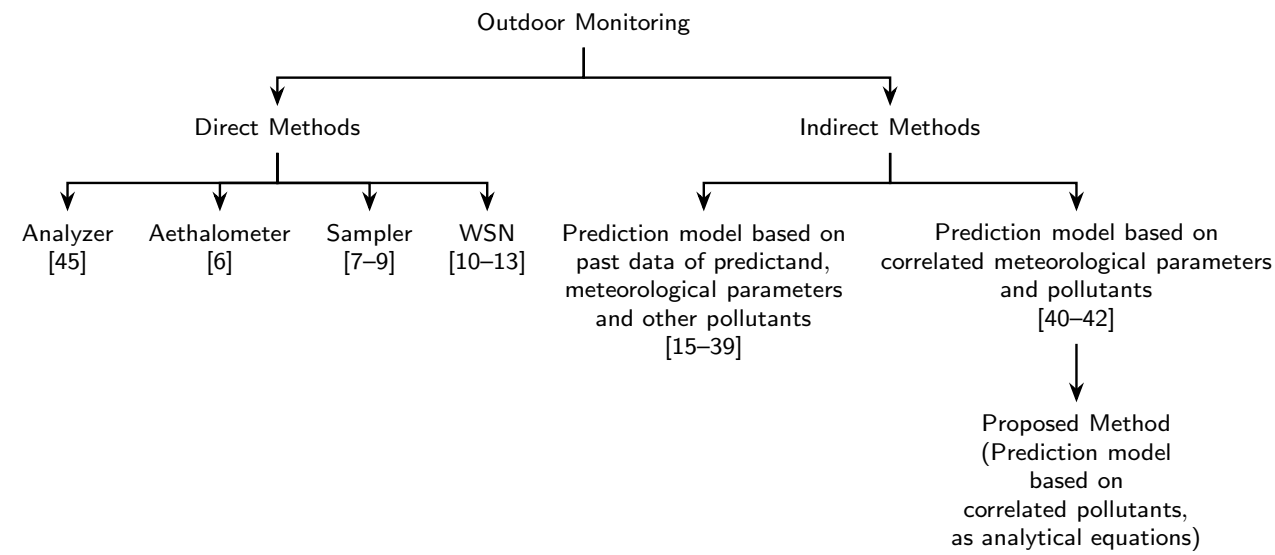


forward neural network (FFNN) with rolling mechanism (RM) and accumulated generating operation (AGO) of gray model (RM-GM-FFNN) was developed [25] for prediction of PM2.5 and PM10 using past data of PM2.5 and PM10 in addition to meteorological data. Prediction of PM2.5 based on the back propagation (BP) neural network was explored [26] using satellite-based aerosol optical depth (AOD), meteorological data and past PM2.5 data. The optimized version of the BP network using a genetic algorithm is proposed in [27].

Few researchers have also explored the hybrid model approach for prediction. A hybrid approach based on the autoregressive and nonlinear model for prediction of $\mathrm{NO}_{2}$ is proposed in [28]. A hybrid approach based on autoregressive integrated moving average model (ARIMA) and ANN is discussed in [29]. The comprehensive forecasting model (CFM) was developed based on ARIMA, ANN and exponential smoothing method (ESM) [30]. Another cluster-based hybrid approach using neural network autoregression (NNAR) and the ARIMA model is discussed in [31] for prediction of PM2.5 using past data of PM2.5. A hybridgeneralized autoregressive conditional heteroskedasticity based prediction approach proposed in [32] for prediction of PM2.5. In [33] hybrid model was built for PM2.5 by applying the trajectory-based geographic model and wavelet transformation into the MLP type of neural network. In which meteorological forecasts and pollutants were used as predictors. Comparison of a hybrid model consisting of an ensemble empirical mode decomposition and general regression neural network (EEMD-GRNN), adaptive neuro-fuzzy inference system (ANFIS), principal component regression (PCR), and MLR is discussed in [34] with best results obtained for EEMD-GRNN model. In [35] multi-task learning framework is used for the prediction of air pollutants which reduces model parameters with improved performance. Convolutional generalization model implemented [36] for prediction of PM2.5 using meteorological data shows mean squared error (MSE) of $15.0 \mu \mathrm{g} / \mathrm{m}^{3}$. A deep learning-based prediction approach is also implemented for prediction using current and/or previous air pollutants and meteorological data [37-39].

The research work in [40] focused on Cuckoo searchleast squares support vector machine (CS-LSSVM) based prediction approach for PM2.5 using correlation and principal component analysis. Previous data of PM2.5 was used as one of the predictors in addition to correlated parameters. The correlation analysis of PM2.5 to other meteorological parameters and pollutants using multivariate statistical analysis method and ANN was implemented in [41] and prediction results show RMSE of $24.06 \mu \mathrm{g} / \mathrm{m}^{3}$ for ANN-based model. Performance comparison of machine learning approaches such as random forests (RF), Support Vector Machines (SVMs) and ANN is presented in
[42]. Furthermore, a calibration model is developed using ANN for black carbon in which meteorological parameter and other correlated pollutants are used as predictors. The lower RMSE and $R^{2}$ closeness to 1 , also showed the effectiveness of the ANN.

Though different methods and instruments for monitoring PM2.5 exist [43], only a few are used for real-time measurement and monitoring. These instruments for PM2.5 lack portability and exhibit a slower response time [44]. Furthermore, the standard procedure is challenging for real-time monitoring and corrective actions $[45,46]$. This is because analyzed data is a delayed response of the current data. Hence real-time monitoring of PM2.5 can be useful. In this work, we propose a method to address the issue of the delayed response of data. We propose the PM2.5 prediction model based on analytical equations, which can be ported to a standard WSN. We envisage that such a method not only provides benefits for real-time monitoring but also enables an existing WSN to extend its capabilities from monitoring to analyzing.

This paper is organized as follows. Section 2 presents the proposed solution for PM2.5 prediction, starting with a general overview of the proposed approach and details about correlation study in Sect. 2.1, followed by the details about the prediction model using ANN in Sect. 2.2. The derived analytical equation for prediction and related results are discussed in Sect. 3. At last, Sect. 4 concludes this paper and gives details about possible future work.

\section{Materials and methods}

The previously developed prediction models based on past data of predictand do not eliminate the need for dedicated instruments and in almost all cases the proprietary tools are used to measure the predictand. This presents an opportunity for developing a correlation-based prediction model in the form of analytical equations. The advantage of such a method results in deployment of a low cost, lightweight and adaptable hardware, as discussed in [47]. Additionally, this hardware "Internet of things enabled low power environmental monitoring system-(IoT-LPEMS)" has shown to be useful for computing the analytical equations for PM2.5 prediction. The ANN is adopted in our work, due to its superior performance discussed in [19-24, 42]. Primary results related to the comparison of SVM and ANN are discussed in the results section and demonstrate the effectiveness of our proposed method.

The contribution of the work is as follows:

1. The study related to correlation among the pollutants and correlation with PM2.5 is performed which found beneficial for deciding predictors and future work. 
2. Analytical equations are proposed for prediction of PM2.5 using ANN.

3. Recalibration of the derived prediction model in terms of coefficients and number of predictors is done to evaluate its performance.

The ANN consists of interconnected computing elements known as neurons with inputs and outputs. As shown in Fig. 2, neuron has $P$ inputs each with weight $W$. The sum $(S)$ of weighted inputs and bias $(b)$ is fed to the transfer function block ( $f$ ). The output of each neuron is obtained by subsequently applying the transfer function to the sum of weighted inputs and bias. The proposed PM2.5 prediction model using the ANN is derived using the steps shown in Fig. 3.

\subsection{Observed data and correlation}

We have performed a study to obtain a correlation between PM2.5 and other pollutants whose results play a prime role in deciding predictors of the proposed model. Thirteen different parameters were collected for the proposed study from a CPCB online station, India ( $\mathrm{N} 23^{\circ}$ $\left.0^{\prime} 16.6287, \mathrm{E} 72^{\circ} 35^{\prime} 48.7816\right)$ that included pollutants; $\mathrm{CO}, \mathrm{NO}, \mathrm{NO}_{2}, \mathrm{SO}_{2}, \mathrm{O}_{3}$, VOC (benzene, toluene, ethyl benzene, $\mathrm{M}+\mathrm{P}$ xylene, $\mathrm{O}$-xylene), PM2.5 and meteorological parameters; temperature and humidity. The dataset was collected for 41 months at a sampling rate of one hour and had a total of 29,928 observations. Finally, these 18,880 observations were available simultaneously for all 13 parameters after removing maintenance data for each of the parameters. The moving average filter was implemented in Matrix Laboratory (MATLAB) and used for removing outliers and smoothing the data. After smoothing, the first 500 results were removed, because the optimum window size for smoothing was found to be 500 . The resultant smoothed data set of 18,380 observations was considered as the golden standard data for developing the prediction model. The original and smoothed data for all 13 parameters are shown in Figs. 4, 5, 6 and 7.

The correlation between any two parameters $x$ and $y$ is represented by the correlation coefficient $R$ as per Eq. (1).

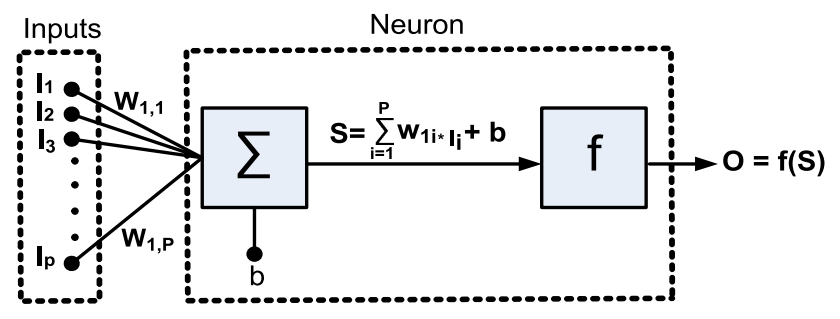

Fig. 2 The structure of the ANN's neuron

$$
R=\frac{n\left(\sum x y\right)-\left(\sum x\right)\left(\sum y\right)}{\sqrt{\left(n \sum x^{2}-\left(\sum x\right)^{2}\right) *\left(n \sum y^{2}-\left(\sum y\right)^{2}\right)}}
$$

where $n$ is the total number of samples. $R$ takes values in the range of $[-1$ to +1$]$. The value of $R$ close to +1 confirms strong positive correlation between $x$ and $y$. For the practical purpose, correlation greater than 0.8 is considered as being strong and less than 0.5 as weak [49].

Correlation between PM2.5 and other parameters in addition to the correlation among the parameters was evaluated using $18 \mathrm{k}+$ data over 41 months. The last column of correlation study results shown in Table 1 conveys the highest correlation of PM2.5 with $\mathrm{NO}_{1} \mathrm{NO}_{2}$, and Benzene and low correlation with temperature and humidity. At typical ambient concentrations, $\mathrm{NO}$ is not considered to be hazardous while $\mathrm{NO}_{2}$ can be hazardous [50]. Furthermore, $\mathrm{NO}_{2}$ is considered as one of the major pollutants by world health organizations and environmental agencies [51, 52]. Hence, $\mathrm{NO}_{2}$ is considered as one of the predictors for the proposed model and $\mathrm{NO}$ is excluded. The results indicate the strong correlation ( $>0.8$ ) of $\mathrm{CO}, \mathrm{NO}_{2}, \mathrm{SO}_{2}$, and VOC (benzene, toluene, ethyl benzene, $\mathrm{M}+\mathrm{P}$ xylene, O-xylene) with PM2.5. These results found useful in deciding the predictors of the proposed PM2.5 prediction model and future research work.

The proposed prediction model is based on supervised learning, where, both inputs and target values are provided as a training dataset. So, for the proposed PM2.5 prediction model, values of input pollutants $\left(\mathrm{CO}, \mathrm{NO}_{2}\right.$, $\mathrm{SO}_{2}$, and $\mathrm{VOC}$ (benzene, toluene, ethyl benzene, $\mathrm{M}+\mathrm{P}$ xylene, O-xylene)) and target (PM2.5) are taken as a training dataset. It is observed that the training of the ANN will be efficient if each parameter of the training dataset is normalized within the range [-1:1]. Normalization of the parameters is done by finding the value of each parameter within the normalized range using Eq. (2).

$x_{n}=\frac{\left(Y_{\max }-Y_{\min }\right) *\left(X-X_{\min }\right)}{\left(X_{\max }-X_{\min }\right)}+Y_{\min }$

where $X$ is the value of the parameter while $X_{\max }$ and $X_{\min }$ are the maximum and minimum values of the parameter, respectively. For example, in CO data, $X$ is the smoothed value of $\mathrm{CO}$ while $\mathrm{X}_{\max }$ and $\mathrm{X}_{\min }$ are maximum and minimum values of smoothed $C O$ data, respectively. Since, normalization range is [-1:1], $Y_{\max }$ is 1 and $Y_{\min }$ is -1 . The normalized parameters can be converted back into their original form using Eq. (3).

$X=\frac{\left(X_{n}-Y_{\min }\right) *\left(X_{\max }-X_{\min }\right)}{\left(Y_{\max }-Y_{\min }\right)}+X_{\min }$

where $x_{n}$ represents normalized data and $X$ is the smoothed data. $X_{\max }$ and $X_{\min }$ are the maximum and

SN Applied Sciences 
Fig. 3 Steps followed for developing the prediction model of PM2.5

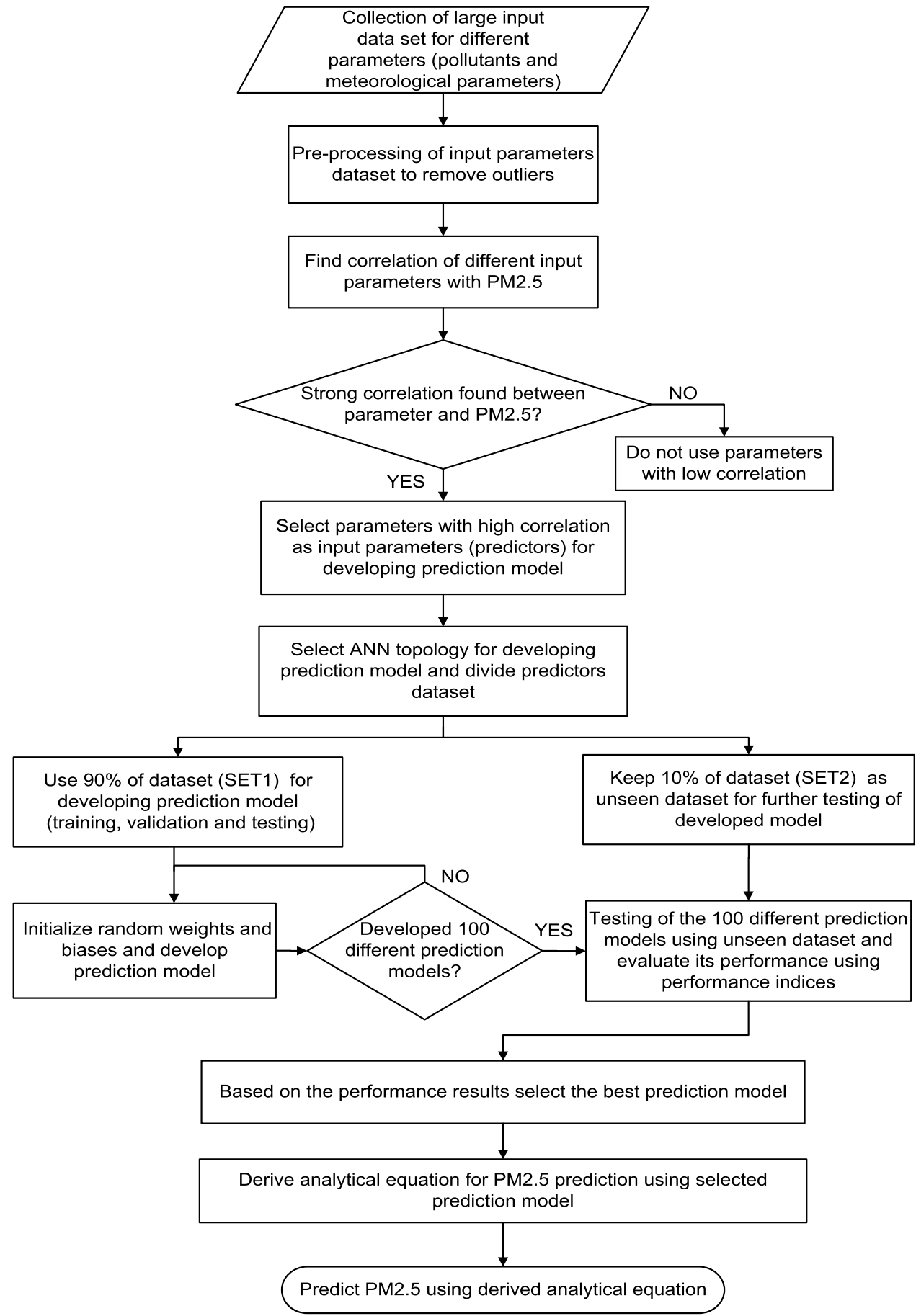

minimum values of smoothed data, respectively. For example, to convert the normalized data of predictand PM2.5 into original form, $X_{\max }$ and $X_{\min }$ values of targeted PM2.5, shown in Fig. 7 are used.

\subsection{Prediction model of PM2.5}

The proposed prediction model of PM2.5 using ANN is shown in Fig. 8. It is based on a feed-forward neural network with a single hidden layer. In the proposed prediction model inputs are selected based on the correlation results discussed in the previous section. Therefore, out of 12 parameters 8 highly correlated (correlation > 0.8) parameters; $\mathrm{CO}, \mathrm{NO}_{2}, \mathrm{SO}_{2}$, and $\mathrm{VOC}$ (benzene, toluene, ethyl benzene, $\mathrm{M}+\mathrm{P}$ xylene, $\mathrm{O}$-xylene) are selected for developing the PM2.5 prediction model. Moreover, the reduction in the number of parameters from 12 to 8 decreases the computation cost of the prediction model. 


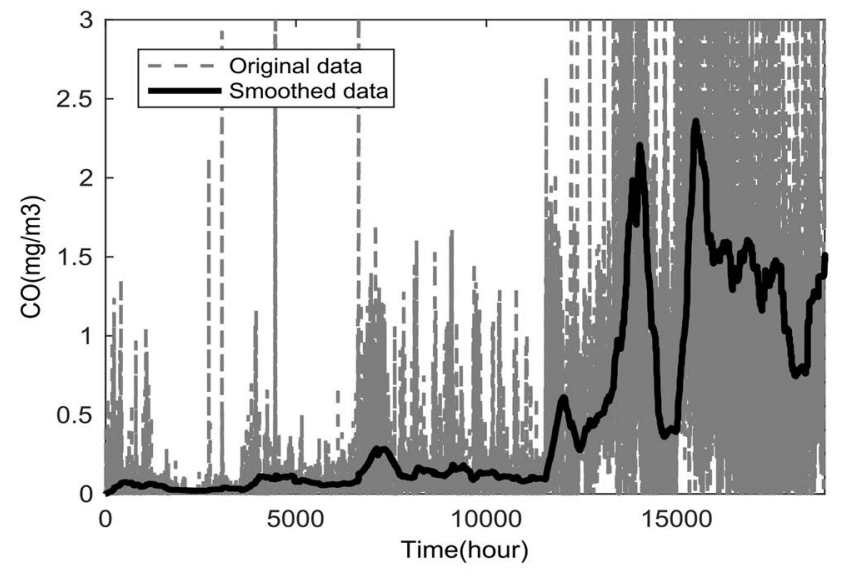

(a) CO Data

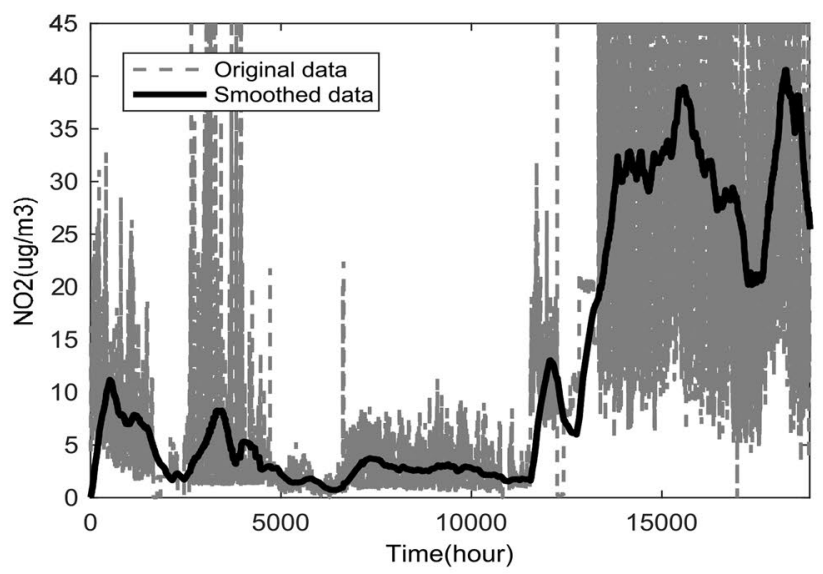

(c) $\mathrm{NO}_{2}$ Data

Fig. 4 Original and smoothed data of $\mathrm{CO}, \mathrm{NO}$ and $\mathrm{NO}_{2}$ and $\mathrm{SO}_{2}$

Selected eight pollutants are the input parameters for each neuron of the hidden layer which consists of eight neurons.

The weights of the hidden layer and output layer can be expressed by a matrix of size $S \times P$, where, $S$ is equal to the number of neurons in the layer and $P$ is equal to the number of inputs of the layer. In our case, the matrix size is $8 \times 8$, as both the inputs and hidden layer size are 8 . The matrix size for the output layer is $1 \times 8$ as it consists of one neuron and eight inputs coming from the hidden layer. For the proposed predicted model, weights of the hidden layer and output layer are represented by $\mathrm{LW}_{1}$ of (of $8 \times 8$ size) and $L_{2}$ of size (of $1 \times 8$ size) respectively. The element, $L W_{1}(1)$ denotes the first row of $L W_{1}$ matrix, which is formed by the weights of inputs going to the first neuron of the hidden layer. Similarly, $\operatorname{LW}_{2}(1,1)$ stands for first row and first column element of matrix $\mathrm{LW}_{2}$ and so on. Biases of the particular layer can be given by a matrix of the size $S \times 1$, where $S$ is equal to the number of neurons in the layer. The bias matrices for hidden layer and output layer

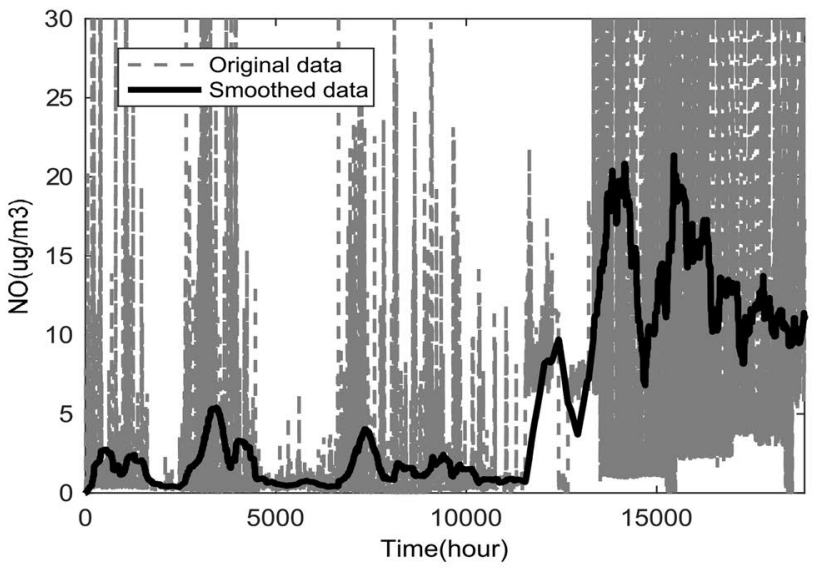

(b) NO Data

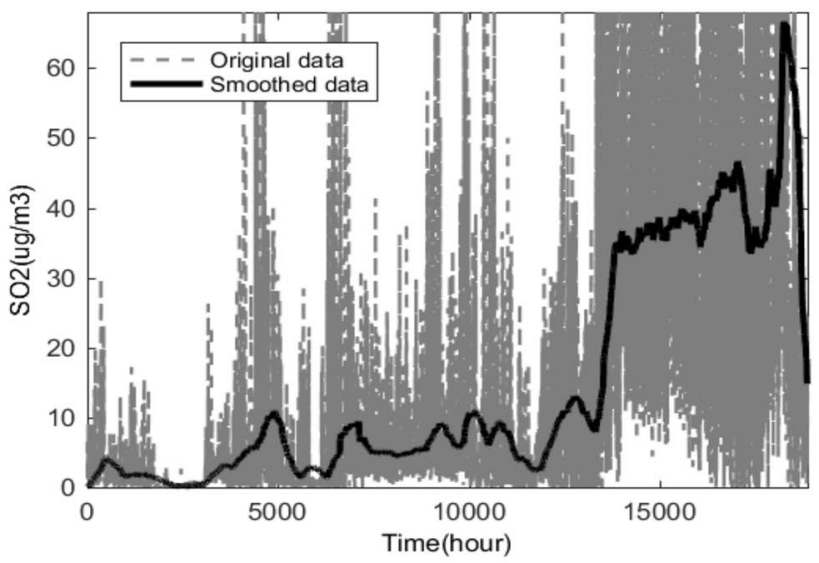

(d) $\mathrm{SO}_{2}$ Data

are $b_{1}$ and $b_{2}$ respectively. As the number of neurons in the hidden layer is 8 and output layer is 1 , the bias matrices, $b_{1}$ is $8 \times 1 a^{a n d} b_{2}$ is $1 \times 1$, where $b_{1}(1,1)$ denotes the first row and first column element of matrix $b_{1}$.

The training function train/m based on the Levenberg-Marquardt algorithm is adopted $[53,54]$ for training. To train the network for the nonlinear relationship between input and output and to constrain output in positive range standard nonlinear transfer function logsig given by Eq. (4) is used in the hidden layer.

$\log \operatorname{sig}(m)=\frac{1}{\left(1+e^{-m}\right)}$

where $m$ is the input to the transfer function. In logsig transfer function, the output will be in the range of [0:1] for the entire range of inputs. For nonlinear regression or prediction, purelin is an effective transfer function for the output layer [55]. Hence in the proposed model, purelin is

\section{SN Applied Sciences}




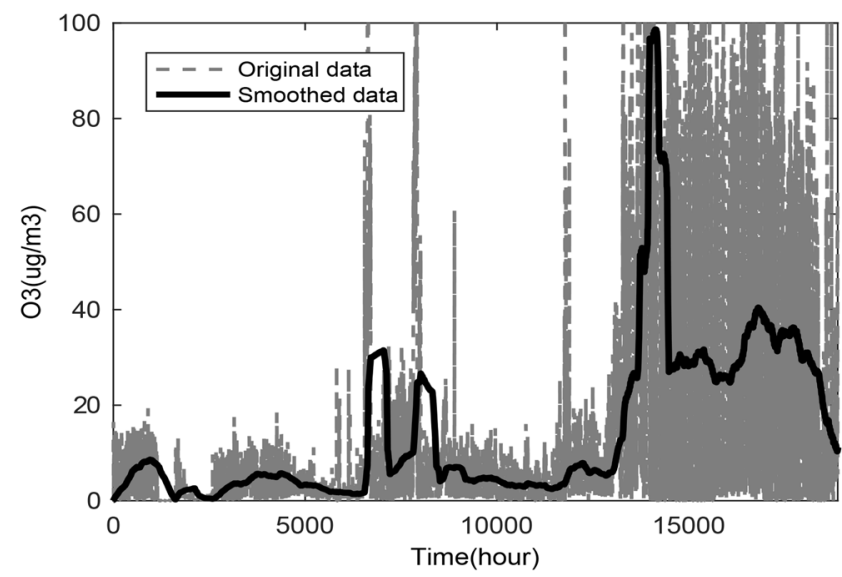

(a) $\mathrm{O}_{3}$ Data

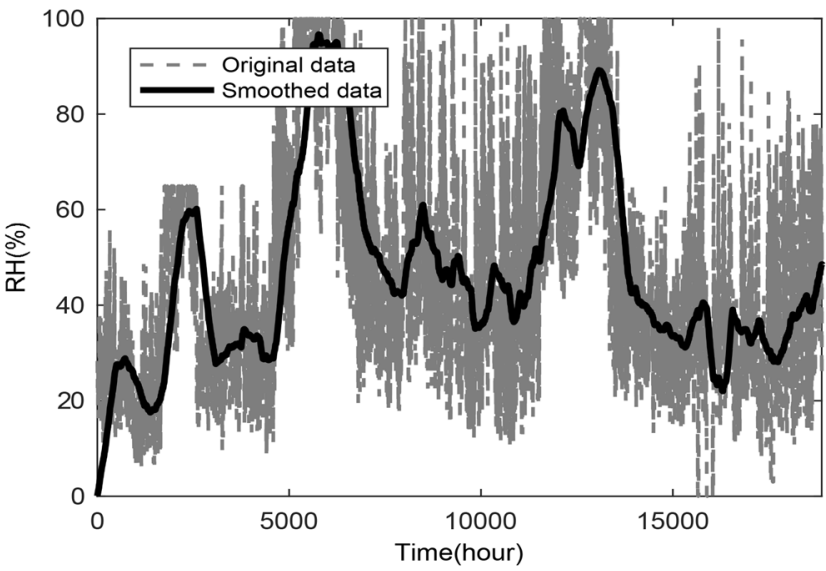

(c) Humidity Data

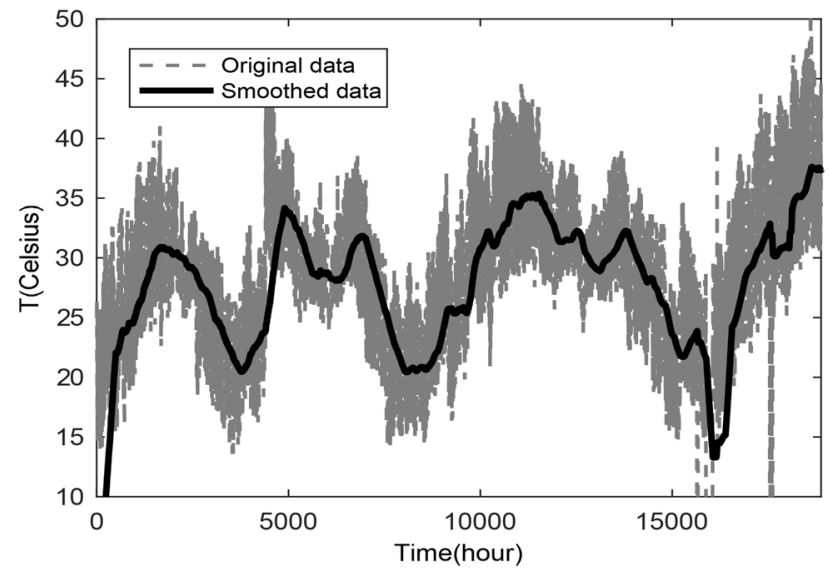

(b) Temperature Data

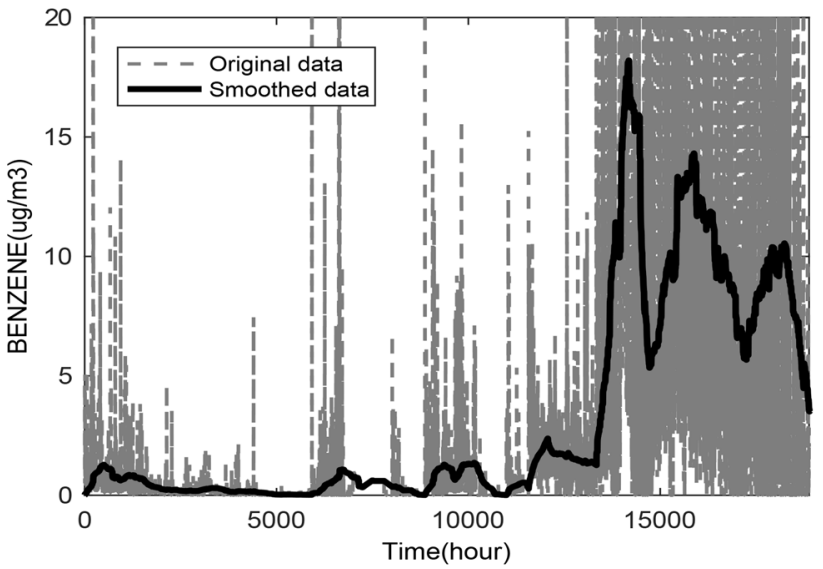

(d) Benzene Data

Fig. 5 Original and smoothed data of $\mathrm{O}_{3}$, temperature, humidity and benzene

used as a transfer function in the output layer. In purelin, the output will be equal to the input.

The total available data of $18 \mathrm{k}+$ is divided into two sets the first set (SET1) includes $90 \%$ of data and the second set (SET2) includes $10 \%$ of data. The division into two sets is done randomly so that all types of data can be included in two sets. The SET1 ( $90 \%$ data) is used for developing the prediction model and it is divided in a standard manner widely used by researchers, $70 \%$ for training, $15 \%$ for validating, and $15 \%$ for testing. The SET2 (10\% data) is kept as unseen data for further testing and comparing the performance of the prediction model.

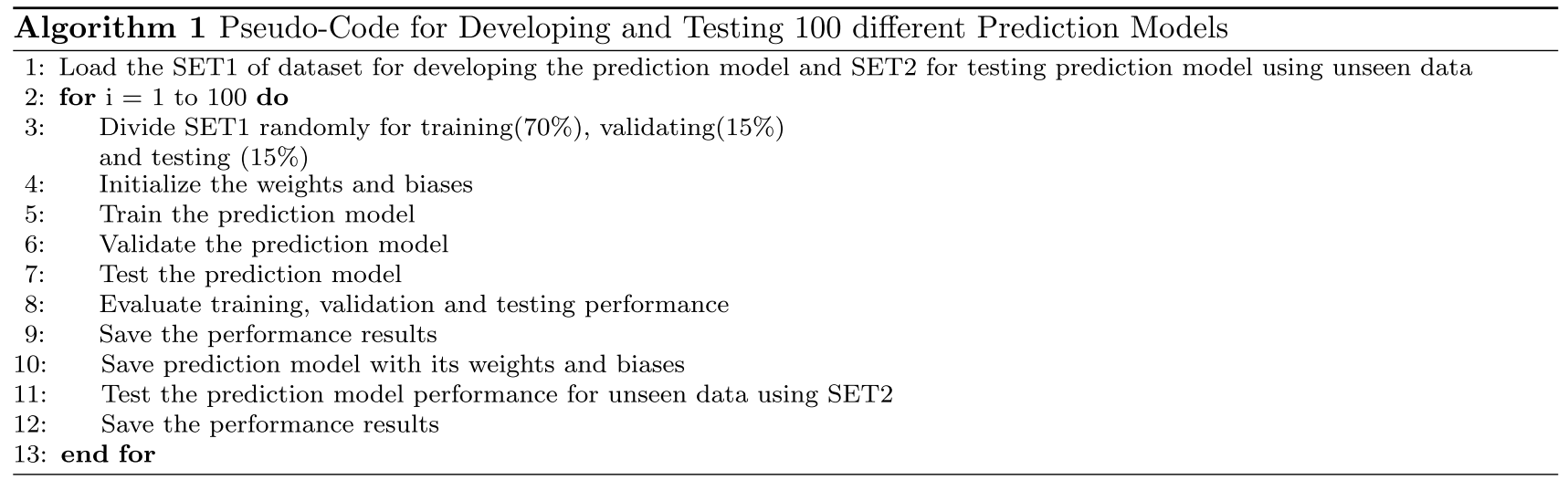




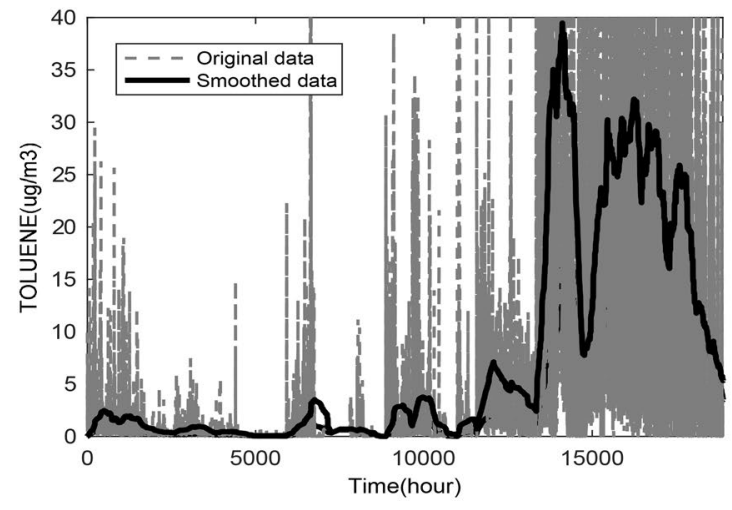

(a) Toluene Data

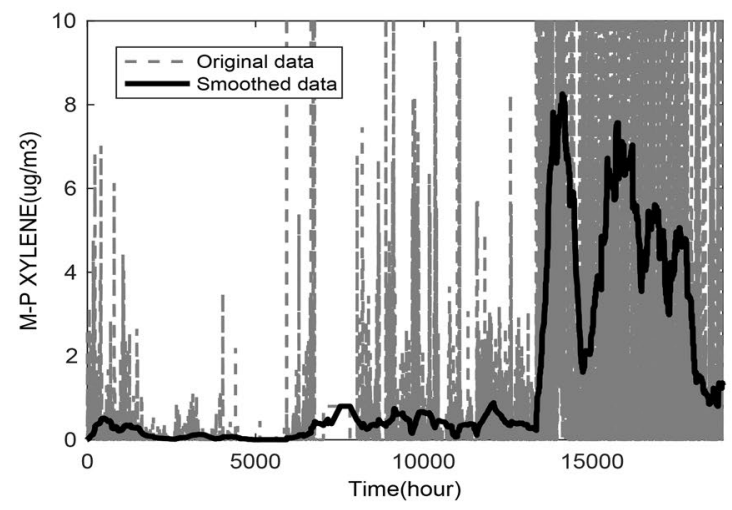

(c) M-P Xylene Data

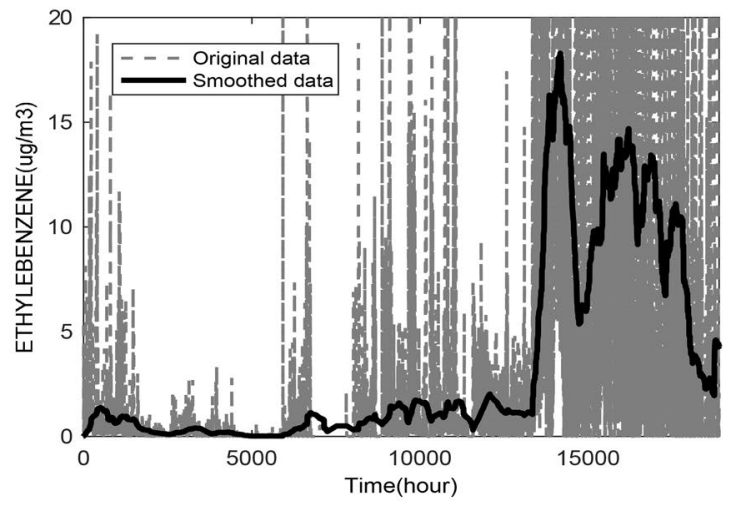

(b) Ethyl Benzene Data

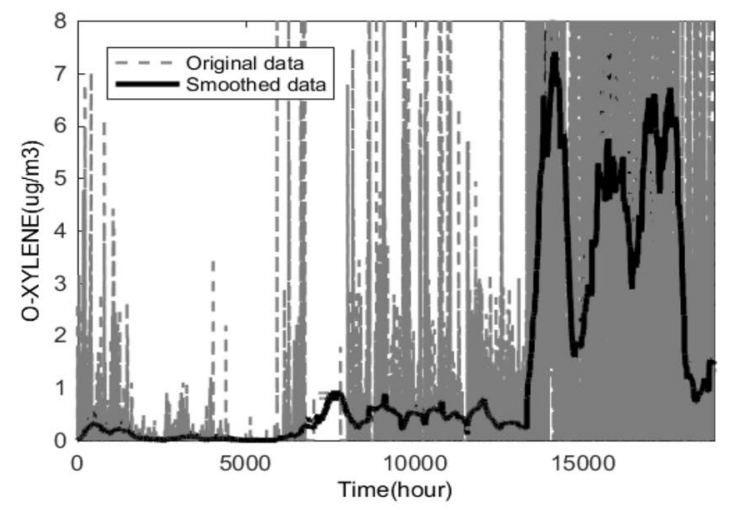

(d) O-Xylene Data

Fig. 6 Original and smoothed data of toluene, ethyl benzene, M-P xylene and O-xylene

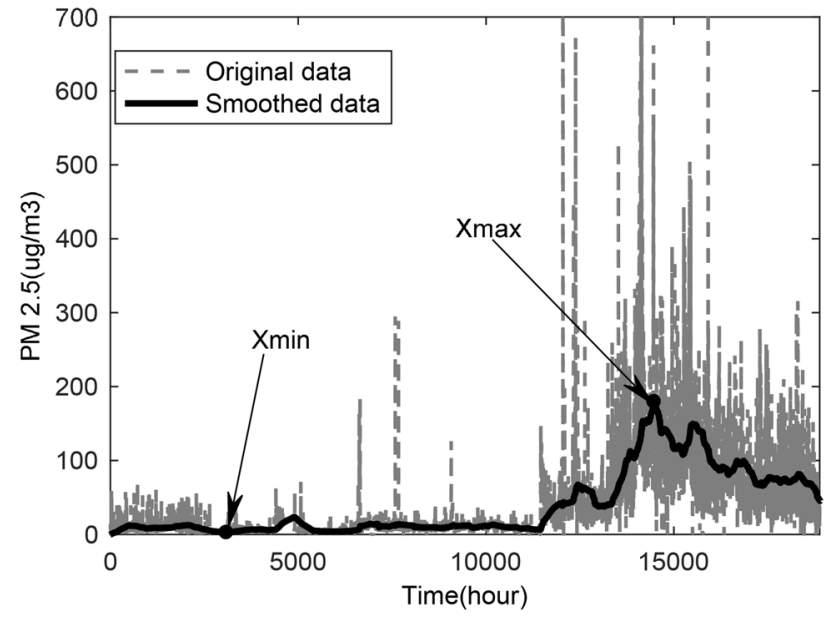

Fig. 7 PM2.5 data

To select the best prediction model, 100 different prediction models are developed and tested as per the pseudo-code in Algorithm 1. This algorithm is repeated 100 times to get the performance of 100 different prediction models for comparing training and testing results with good generalization. The performance of each prediction model is evaluated based on, RMSE and $R^{2}$, where, RMSE is the root mean square of the errors, i.e, the difference between the target value and the predicted value and is given in Eq. (5).

$R M S E=\sqrt{\frac{\sum_{i=1}^{n}\left(A_{i}-P_{i}\right)^{2}}{n}}$

where $A_{i}$ represents the actual value, $P_{i}$ represents the predicted value and $n$ is the total number of samples. $R^{2}$ is the coefficient of determination and it is the square of the correlation $\mathrm{R}$ (Eq. 1). The closeness between target values and predicted outputs of the prediction model is represented by $R^{2}$. The value of $R^{2}$ equal to 1 represents targets and predicted outputs are very close to each other. The best prediction model is selected from 100 different prediction models based on these performance indices. The performance indices results of the selected model are presented in the next section. Furthermore, the derived analytical equation for PM2.5 prediction using the selected prediction model is also discussed in the next section with promising results. 
Table 1 Correlation between different parameters found through correlation study

\begin{tabular}{llllllllllllll}
\hline & $\mathrm{CO}$ & $\mathrm{NO}$ & $\mathrm{NO}_{2}$ & $\mathrm{SO}_{2}$ & $\mathrm{O}_{3}$ & $\mathrm{~T}$ & $\mathrm{RH}$ & $\mathrm{Ben}$ & $\mathrm{Tol}$ & Eth & $\mathrm{M}$-xyl & $\mathrm{O}-\mathrm{xyl}$ & $\mathrm{PM} 2.5$ \\
\hline $\mathrm{CO}$ & 1 & 0.94 & 0.87 & 0.83 & 0.77 & -0.07 & -0.22 & 0.90 & 0.93 & 0.91 & 0.92 & 0.89 & $\mathbf{0 . 8 3}$ \\
$\mathrm{NO}$ & 0.94 & 1 & 0.93 & 0.83 & 0.79 & -0.12 & -0.23 & 0.93 & 0.93 & 0.91 & 0.91 & 0.86 & $\mathbf{0 . 9 1}$ \\
$\mathrm{NO}_{2}$ & 0.87 & 0.93 & 1 & 0.93 & 0.73 & -0.08 & -0.31 & 0.92 & 0.85 & 0.83 & 0.81 & 0.77 & $\mathbf{0 . 9 1}$ \\
$\mathrm{SO}_{2}$ & 0.83 & 0.83 & 0.93 & 1 & 0.71 & 0.01 & -0.33 & 0.88 & 0.82 & 0.79 & 0.77 & 0.77 & $\mathbf{0 . 8 5}$ \\
$\mathrm{O}_{3}$ & 0.77 & 0.79 & 0.73 & 0.71 & 1 & -0.07 & -0.24 & 0.84 & 0.85 & 0.85 & 0.83 & 0.83 & 0.77 \\
$\mathrm{~T}$ & -0.07 & -0.12 & -0.08 & 0.01 & -0.07 & 1 & 0.29 & -0.13 & -0.16 & -0.19 & -0.22 & -0.13 & -0.08 \\
$\mathrm{RH}$ & -0.22 & -0.23 & -0.31 & -0.33 & -0.24 & 0.29 & 1 & -0.32 & -0.30 & -0.32 & -0.32 & -0.32 & -0.22 \\
Benzene & 0.90 & 0.93 & 0.92 & 0.88 & 0.84 & -0.13 & -0.32 & 1 & 0.96 & 0.94 & 0.93 & 0.89 & $\mathbf{0 . 9 2}$ \\
Toluene & 0.93 & 0.93 & 0.85 & 0.82 & 0.85 & -0.16 & -0.30 & 0.96 & 1 & 0.99 & 0.98 & 0.96 & $\mathbf{0 . 8 8}$ \\
Ethyl benzene & 0.91 & 0.91 & 0.83 & 0.79 & 0.85 & -0.19 & -0.32 & 0.94 & 0.99 & 1 & 0.99 & 0.96 & $\mathbf{0 . 8 8}$ \\
M-P Xylene & 0.92 & 0.91 & 0.81 & 0.77 & 0.83 & -0.22 & -0.32 & 0.93 & 0.98 & 0.99 & 1 & 0.96 & $\mathbf{0 . 8 6}$ \\
O-Xylene & 0.89 & 0.86 & 0.77 & 0.77 & 0.83 & -0.13 & -0.32 & 0.89 & 0.96 & 0.96 & 0.96 & 1 & $\mathbf{0 . 8 2}$ \\
PM2.5 & 0.83 & 0.91 & 0.91 & 0.85 & 0.77 & -0.08 & -0.22 & 0.92 & 0.88 & 0.88 & 0.86 & 0.82 & 1 \\
\hline
\end{tabular}

A high correlation more than 0.8 is highlighted

Fig. 8 The ANN topology used for developing PM2.5 prediction model
Input
Hidden
Output
layer
layer
layer

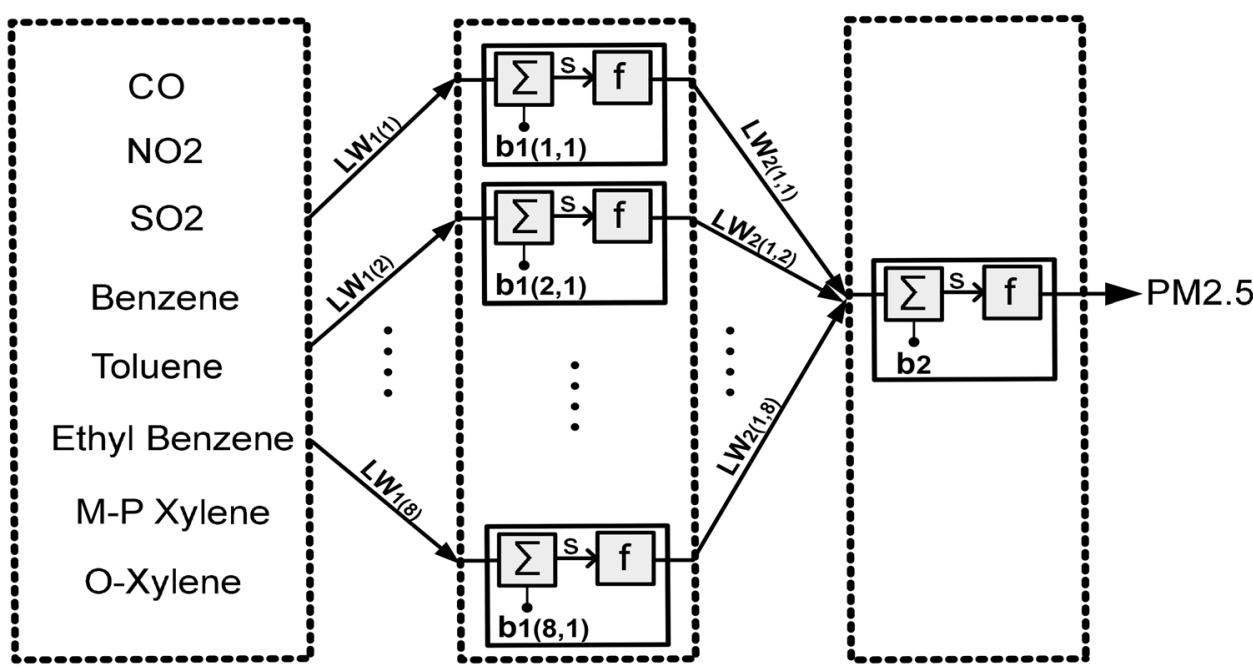

Table 2 Performance results of SVM and ANN

Table 3 Performance results of the proposed PM2.5 prediction model

\begin{tabular}{lll}
\hline Performance of & RMSE & $R^{2}$ \\
\hline Training & 1.61 & 0.99 \\
Validation & 1.63 & 0.99 \\
Testing & 1.58 & 0.99 \\
Testing for unseen data & 1.51 & 0.99 \\
\hline
\end{tabular}

\section{Results and discussion}

In this section, computing and comparison results are provided to demonstrate the effectiveness of the proposed approach. The RMSE and $R^{2}$ are used as performance indices. In the initial phase of developing the proposed prediction model, the performance of ANN was compared with SVM. The comparison results shown Table 2 demonstrates RMSE of 2.82 and 1.51 during testing for SVM and ANN respectively. Hence, ANN is used for developing the proposed prediction model.

One of the problems that occur during developing prediction model using ANN is overfitting. The overfitting presence can be detected when the error for the training dataset is very small, but when new data is presented to 
the prediction model the error is large. This is because the prediction model has memorized the training examples, but it has not learned to generalize to new situations. To develop the proposed prediction model (shown in Fig. 8) with good generalization, Algorithm 1 is used. Table 3 shows the best performance of the prediction model obtained from 100 iterations. The results show RMSE of 1.61 and $R^{2}$ of 0.99 during training and RMSE of 1.51 and $R^{2}$ of 0.99 during testing using unseen data. It is visible from the results that very little variation between training and testing errors, which confirms good generalization without overfitting. The results demonstrate the closeness of the predicted values with the target values or actual values, so this prediction model is used further to derive an analytical equation for PM2.5 prediction.

The hidden layer and output layer weights of the proposed prediction model are specified by Eqs. (6) and (7) respectively. The hidden layer and output layer biases of the proposed prediction model are given by Eqs. (8) and (9) respectively. Matrix $B_{1}$ is formed by repeating the hidden layer bias matrix $\mathrm{b}_{1}, \mathrm{~N}$ times, where $\mathrm{N}$ is equal to the size of test dataset (1838 in our case). This operation shown in Eq. (10), is performed to make bias matrix size $B_{1}$ equal to the size of product term matrix $L W_{1}^{*} x_{n}$, so that addition operation $\left(B_{1}+L W_{1}^{*} x_{n}\right)$, shown in Eq. (11) can be performed.

$$
L W_{1}=\left[\begin{array}{ccccccccc}
-25.98 & -31.06 & 17.51 & 38.45 & -37.35 & 97.71 & -92.95 & 65.96 \\
3.30 & 6.46 & 0.20 & -1.98 & 10.08 & -10.28 & 3.35 & -3.78 \\
-2.66 & 2.50 & -1.08 & -1.01 & -0.19 & 1.70 & -2.77 & 1.69 \\
-6.07 & 6.14 & -2.21 & -3.59 & 1.80 & -3.00 & 0.22 & 2.80 \\
-9.79 & -7.75 & -4.42 & 27.23 & -4.41 & 0.02 & -6.28 & 8.46 \\
3.82 & -6.23 & 1.24 & -1.45 & 5.60 & 1.85 & 1.80 & -5.86 \\
3.15 & 6.32 & 0.37 & -0.83 & 10.75 & -11.22 & 2.65 & -3.43 \\
-2.13 & 1.99 & -1.12 & -0.03 & 0.04 & 4.09 & -4.88 & 1.33
\end{array}\right]
$$

Finally, the derived analytical equation for PM2.5 prediction is represented by Eq. (11).

$P M 2.5_{n}=b_{2}+L W_{2} * \log \operatorname{sig}\left(B_{1}+L W_{1} * x_{n}\right)$

where PM2.5 $5_{n}$ is normalized output. The input matrix $\mathrm{x}_{n}$ is formed using normalized values of $\mathrm{CO}, \mathrm{NO}_{2}, \mathrm{SO}_{2}$, and VOC and the size of $x_{n}$ is $P \times N$ where $P=8$ is the number of inputs and $N=1838$ is the number of values for each input. The normalized value of PM2.5 is converted back into original form using Eq. (12) which is derived using Eq. (3) in which $X_{\max }$ and $X_{\min }$ are taken as per Fig. 7.

$P M 2.5=\frac{\left(P M 2.5_{n}+1\right) *(180.052-2.228)}{2}+2.228$

To prove the effectiveness of the proposed approach, Eqs. (11) and (12) are computed using excel sheet (lowcost processing tool) as shown in Fig. 9. The matrix $x$ of size $8 \times 1838$ is taken as input matrix, which represents the testing data set. The proposed prediction equations are computed through following steps.

a. First, $x_{n}$ is obtained by computing Eq. (2).

b. Multiplying the two matrices of $\mathrm{LW}_{1}$ (Eq. 6) and $\mathrm{x}_{n}$, product term $\mathrm{LW}_{1}{ }^{*} x_{n}$ is obtained.

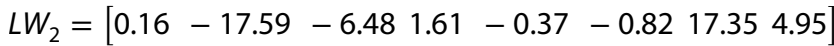

$b_{1}=\left[\begin{array}{c}34.47 \\ 4.00 \\ -1.02 \\ -2.62 \\ 5.70 \\ -0.15 \\ 4.38 \\ -1.96\end{array}\right]$

$b_{2}=[1.59]$

$B_{1}=\left[\begin{array}{lllllll}b_{1} & b_{1} & b_{1} & b_{1} & \ldots & \ldots N\end{array}\right]$
C. Matrix $B_{1}$, formed using Eq. (10) is added in the product term $\mathrm{LW}_{1}^{*} \mathrm{x}_{n}$.

d. Hidden layer activation function (Eq. 4) is applied to find the output of the hidden layer.

e. The output of the hidden layer is multiplied by $\mathrm{LW}_{2}$ (Eq. 7).

f. Then matrix $b_{2}$ from Eq. (9), is added.

Finally, PM2.5 (marked as (A) in Fig. 9) is obtained by computing Eq. (11), which is the normalized value of PM2.5. PM2.5 in the original unit is obtained by processing Eq. (12) (marked as (B) in Fig. 9). Predicted values of PM2.5 obtained from Eq. (12) (marked as (B) in Fig. 9) are close to the actual values of PM2.5 (marked as (C) in Fig. 9). These results are plotted in Fig. 10, which again confirms 
Fig. 9 Excel sheet used for computing PM2.5 prediction model equation
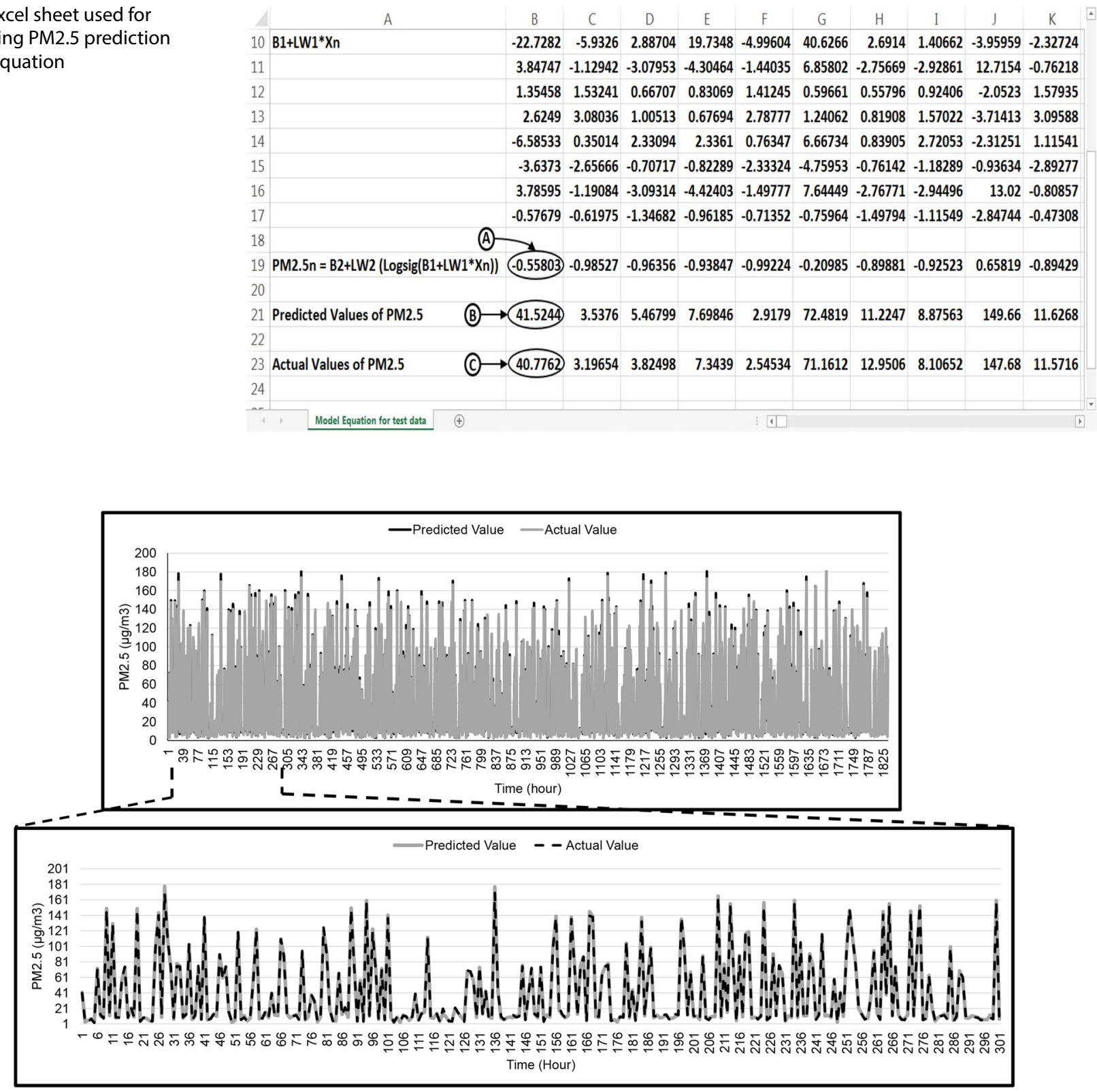

Fig. 10 The good match achieved between predicted values and actual values of PM2.5 using derived analytical equation for prediction

the closeness of predicted values with actual values. The obtained RMSE and $R^{2}$ are $1.80 \mu \mathrm{g} / \mathrm{m}^{3}$ and 0.99 respectively. Hence performance results of derived analytical equation are found in close accordance with prediction model results shown in Table 3. Furthermore, results express the effectiveness of the derived analytical equation for environment monitoring using low-cost processing tools and/or WSN.

Previously developed prediction models, depend on past data, are often time-consuming and use dedicated instruments. In the prediction model, we have eliminated the above requirements and comparison with some researches is shown in Table 4. As can be seen, the proposed approach has better RMSE and $R^{2}$ compared to existing methods. The RMSE of $1.80 \mu \mathrm{g} / \mathrm{m}^{3}$ and $R^{2}$ of 0.99 is obtained for the test dataset of size 1838 . We extended the model to accommodate a reduced test dataset of size 10 , which shows RMSE of $146.10 \mu \mathrm{g} / \mathrm{m}^{3}$ and $R^{2}$ of 0.95 . This performance of the prediction approach is due to the dependence of RMSE on the number of samples or test data set size which can be optimized depending on the availability of computing data for prediction. 
Table 4 The summary of performance results obtained for previously developed prediction models and the proposed analytical equation for prediction

\begin{tabular}{llcl}
\hline References & Predictand & RMSE & $R^{2}$ \\
\hline$[19]$ & $\mathrm{O}_{3}(\mathrm{ppb})$ & 0.30 & 0.69 \\
{$[20]$} & $\mathrm{O}_{3}\left(\mu \mathrm{g} / \mathrm{m}^{3}\right)$ & 21.78 & 0.73 \\
{$[21]$} & $\mathrm{NO}_{2}(\mathrm{ppb})$ & 7.3 & 0.91 \\
{$[28]$} & $\mathrm{NO}_{2}\left(\mu \mathrm{g} / \mathrm{m}^{3}\right)$ & 13.93 & 0.93 \\
{$[22]$} & $\mathrm{PM} \mathrm{10}\left(\mu \mathrm{g} / \mathrm{m}^{3}\right)$ & 12.16 & 0.83 \\
{$[29]$} & $\mathrm{PM} \mathrm{10}\left(\mu \mathrm{g} / \mathrm{m}^{3}\right)$ & 11.66 & 0.983 \\
{$[26]$} & $\mathrm{PM} 2.5\left(\mu \mathrm{g} / \mathrm{m}^{3}\right)$ & 41.97 & - \\
{$[30]$} & $\mathrm{PM} 2.5\left(\mu \mathrm{g} / \mathrm{m}^{3}\right)$ & 12.89 & - \\
{$[25]$} & $\mathrm{PM} 10\left(\mu \mathrm{g} / \mathrm{m}^{3}\right)$ & 18.4 & 0.90 \\
{$[25]$} & $\mathrm{PM} 2.5\left(\mu \mathrm{g} / \mathrm{m}^{3}\right)$ & 12.7 & 0.95 \\
{$[23]$} & $\mathrm{PM} 2.5\left(\mu \mathrm{g} / \mathrm{m}^{3}\right)$ & 6.77 & 0.99 \\
{$[34]$} & $\mathrm{PM} 2.5\left(\mu \mathrm{g} / \mathrm{m}^{3}\right)$ & 5.03 & 0.79 \\
{$[40]$} & $\mathrm{PM} 2.5\left(\mu \mathrm{g} / \mathrm{m}^{3}\right)$ & 14.47 & - \\
{$[41]$} & $\mathrm{PM} 2.5\left(\mu \mathrm{g} / \mathrm{m}^{3}\right)$ & 24.06 & - \\
{$[42]$} & $\mathrm{BC}\left(\mathrm{ng} / \mathrm{m}^{3}\right)$ & 1480.75 & 0.59 \\
This work & $\mathrm{PM} 2.5\left(\mu \mathrm{g} / \mathrm{m}^{3}\right)$ & 1.80 & 0.99 \\
\hline
\end{tabular}

$L W_{1}=\left[\begin{array}{ccc}26.28 & 3.46 & -12.39 \\ 17.90 & -0.86 & 11.31 \\ -1 & -0.50 & -1.21\end{array}\right]$

$L W_{2}=\left[\begin{array}{lll}-1.01 & 1.38-1.67\end{array}\right]$

$b_{1}=\left[\begin{array}{c}9.93 \\ 21.94 \\ 1.10\end{array}\right]$

$b_{2}=[0.69]$

The proposed prediction model is recalibrated in terms of the number of predictors, weights, and biases to show the fruitfulness of the proposed approach. Instead of eight predictors, three predictors, $\mathrm{CO}, \mathrm{NO}_{2}$, and Benzene (VOC component) are taken considering the availability of low-cost sensors [56] which includes this type of multiple sensing parameters. The proposed approach can work for any other three sensing parameters after recalibrating model. For recalibration prediction model shown in Fig. 8 is used with the same training and testing dataset (of three parameters), but the size of the input layer and the hidden layer is reduced to three. Extracted weights and biases are represented in Eqs. (13) to (16). The performance results shown in Table 5 convey RMSE of $7.54 \mu \mathrm{g} / \mathrm{m}^{3}$ and $R^{2}$ of 0.97 . Hence, the RMSE of the prediction model using three predictors is higher compared to prediction model using
Table 5 Performance results of SVM and ANN

\begin{tabular}{lll}
\hline Performance of & RMSE & $\mathrm{R}^{2}$ \\
\hline Training & 7.93 & 0.97 \\
Validation & 8.10 & 0.97 \\
Testing & 8.00 & 0.97 \\
Testing for unseen data & 7.54 & 0.97 \\
\hline
\end{tabular}

eight predictors but still results are in acceptable range. At the same time, depending on the requirement, it is possible to optimize the accuracy at the cost of increased predictors.

The proposed analytical equation for prediction manifests future usability to monitor $\mathrm{PM} 2.5$ using $\mathrm{CO}, \mathrm{NO}_{2}, \mathrm{SO}_{2}$, and $\mathrm{VOC}$ values only. Additionally, the proposed analytical equation can be computed using any low-cost processing tool or WSN and eliminates the need for costly proprietary tools and analyzers. Therefore, the proposed prediction approach ultimately addresses challenges of existing methodology mentioned earlier such as cost, portability, power consumption, and low tempo-spatial resolution.

The deployment of the developed prediction model needs to consider the following limitations based on the location, available monitoring stations and available monitoring parameters as predictors.

- Air pollution varies from one location to another based on the few parameters like human activity, traffic condition, the structure of urban area and weather conditions. Based on the location, predictors and predictand values as well as their maximum and the minimum limit will change. So, the application of the presented prediction model for another location needs model training, validation, and testing again which provides new coefficients in terms of weights and biases for accurate prediction with lower RMSE.

- Application of prediction model to another location requires a large set of authentic data for training which is sometimes difficult due to the limitation on the number of online environment monitoring stations. Due to a few monitoring parameters, and delay in the availability of data; offline stations are less preferable than online stations.

- Online stations of CPCB monitors pollutants that are greater than the offline stations. Concentration data for a large set of pollutants is the basic requirement for a correlation study or developing a prediction model. Due to the limitation on the online stations available in the city, data were used from only one online station for training, validation, and testing in the proposed study. This can be expanded in the future by taking data from multiple online stations of different cities. 
- The derived prediction model based on ANN generally shows poor performance for predicting the sudden large change in predictors. As, sometimes it is difficult to discriminate between the outliers and sudden changes in the value, applying a smoothing algorithm to remove outliers will also remove the sudden large change in the value of the predictor. This limitation can be targeted through different data processing algorithm and is left as part of future work

\section{Conclusion and future work}

In this paper, we have proposed a novel analytical equation-based prediction approach for PM2.5. The proposed prediction approach adopts ANN as it shows better performance. Moreover, a correlation study is performed using standardized CPCB data which reveals the strong correlation between PM2.5 and eight pollutants. The correlation study results found effective in determining relevant predictors of the prediction model as well as for future research work. The computing results of the analytical equation using excel sheet demonstrates the proximity of predicted and actual results. Furthermore, the proposed analytical equation for the prediction can be computed by any low-cost processing tool or WSN which eliminates the need for costly proprietary tools and analyzers. The results of the recalibrated model for three predictors confirms the implementation using minimum power-hungry gas sensors on the WSN. The comparison results have verified the prominent performance of the proposed prediction approach in terms of performance metrics which proves its capability to overcome the challenges of existing methodology. In addition to this, the proposed approach facilitates an efficient method for online and offline monitoring of PM2.5 with a minimum computational cost. The obtained results can be improved in the future by recalibrating prediction model based on the data available from multiple stations located at the place of deployment. Moreover, probing deeper we would like to extend this work by the implementation of the proposed analytical equation using the WSN.

Acknowledgements The authors thank the Central Pollution Control Board (CPCB) and their staff for their support during this research work.

\section{Compliance with ethical standards}

Conflict of interest The authors declare that they have no conflict of interest.

\section{References}

1. Central Pollution Control Board (2019) National Air Quality Monitoring Programme. http://cpcb.nic.in/about-namp/. Accessed 10 January 2019

2. Central Pollution Control Board (2018) National Air Quality Index.http://www.indiaenvironmentportal.org.in/files/file/ Air\%20Quality\%20Index.pdf. Accessed 25 December 2018

3. Central Pollution Control Board (2018) How is AQI calculated. https://app.cpcbccr.com/ccr_docs/How_AQI_Calculated.pdf. Accessed 24 September 2018

4. World Health Organization (2018) Health effects of particulate matter. http://www.euro.who.int/__data/assets/pdf_ file/0006/189051/Health-effects-of-particulate-matter-final -Eng.pdf. Accessed 20 December 2018

5. Hodan WM, Barnard WR (2004) Evaluating the contribution of PM2.5 precursor gases and re-entrained road emissions to mobile source PM2.5 particulate matter emissions. MACTEC Federal Programs, Research Triangle Park, NC

6. Vilcassim MR, Thurston GD, Peltier RE, Gordon T (2014) Black carbon and particulate matter (PM2.5) concentrations in New York City's subway stations. Environ Sci Technol 48(24):14738-14745

7. Gehrig R, Buchmann B (2003) characterising seasonal variations and spatial distribution of ambient PM10 and PM2.5 concentrations based on long-term Swiss monitoring data. Atmos Environ 37(19):2571-80

8. Northcross A, Chowdhury Z, McCracken J, Canuz E, Smith KR (2010) Estimating personal PM2.5 exposures using CO measurements in guatemalan households cooking with wood fuel. $J$ Environ Monit 12(4):873-8

9. Querol X, Alastuey A, Rodriguez S, Plana F, Mantilla E, Ruiz CR (2001) Monitoring of PM10 and PM2.5 around primary particulate anthropogenic emission sources. Atmos Environ 35(5):845-58

10. Hall J, Loo SM, Stephenson D, Butler R, Pook M, Kiepert J, Anderson J, Terrell N (2012) A portable wireless particulate sensor system for continuous real-time environmental monitoring. In: 42nd international conference on environmental systems, pp $1-14$

11. Mansour S, Nasser N, Karim L, Ali A (2014) Wireless sensor network-based air quality monitoring system. In: IEEE international conference on computing, networking and communications, pp 545-550

12. Kasar AR, Khemnar DS, Tembhurnikar NP (2013) WSN based air pollution monitoring system. Int J Sci Eng Appl 2(4):55-9

13. Liu JH, Chen YF, Lin TS, Lai DW, Wen TH, Sun $\mathrm{CH}$, Juang JY, Jiang JA (2011) Developed urban air quality monitoring system based on wireless sensor networks. In: IEEE international conference on sensing technology, pp 549-554

14. Yi W, Lo K, Mak T, Leung K, Leung Y, Meng M (2015) A survey of wireless sensor network based air pollution monitoring systems. Sensors 15(12):31392-427

15. Ordieres JB, Vergara EP, Capuz RS, Salazar RE (2005) Neural network prediction model for fine particulate matter (PM2.5) on the US-Mexico Border in El Paso (Texas) and Ciudad Juárez (Chihuahua). Environ Modell Softw 20(5):547-59

16. Hooyberghs J, Mensink C, Dumont G, Fierens F, Brasseur O (2005) A neural network forecast for daily average PM10 concentrations in Belgium. Atmos Environ 39(18):3279-89

17. Lv B, Cobourn WG, Bai Y (2016) Development of nonlinear empirical models to forecast daily PM2.5 and ozone levels in three large Chinese Cities. Atmos Environ 147:209-23 
18. Perez P, Gramsch E (2016) Forecasting hourly PM2.5 in Santiago De Chile with emphasis on night episodes. Atmos Environ 124:22-7

19. Lu HC, Hsieh JC, Chang TS (2006) Prediction of daily maximum ozone concentrations from meteorological conditions using a two-stage neural network. Atmos Res 81(2):124-39

20. Sousa SI, Martins FG, Alvim-Ferraz MC, Pereira MC (2007) Multiple linear regression and artificial neural networks based on principal components to predict ozone concentrations. Environ Model Softw 22(1):97-103

21. Gardner MW, Dorling SR (1999) Neural network modelling and prediction of hourly $\mathrm{NO}_{x}$ and $\mathrm{NO}_{2}$ concentrations in urban air in London. Atmos Environ 33(5):709-19

22. Grivas G, Chaloulakou A (2006) Artificial neural network models for prediction of PM10 hourly concentrations, in the greater area of Athens, Greece. Atmos Environ 40(7):1216-29

23. Chen B, Wang X, Yu L, Wang H, Li Y, Chen J, Zhu J, Nan H, Hou L (2016) Prediction of PM2.5 concentration in a agricultural park based on BP artificial neural network. Adv J Food Sci Technol 11(4):274-80

24. Biancofiore $F$, Busilacchio $M$, Verdecchia $M$, Tomassetti B, Aruffo E, Bianco S, Di Tommaso S, Colangeli C, Rosatelli G, Di Carlo P (2017) Recursive neural network model for analysis and forecast of PM10 and PM2.5. Atmos Pollut Res 8(4):652-9

25. Fu M, Wang W, Le Z, Khorram MS (2015) Prediction of particular matter concentrations by developed feed-forward neural network with rolling mechanism and gray model. Neural Comput Appl 26(8):1789-97

26. Chen $Y$ (2018) Prediction algorithm of PM2.5 mass concentration based on adaptive BP neural network. Computing 100:825-838

27. Wang $X$, Wang $B(2018)$ Research on prediction of environmental aerosol and PM2.5 based on artificial neural network. Neural Comput Appl. https://doi.org/10.1007/s00521-018-3861-y

28. Chelani AB, Devotta $S$ (2006) Air quality forecasting using a hybrid autoregressive and nonlinear model. Atmos Environ 40(10):1774-80

29. Samia A, Kaouther N, Abdelwahed T (2012) A hybrid ARIMA and artificial neural networks model to forecast air quality in urban areas: case of Tunisia. Adv Mater Res 518:2969-2979

30. Liu DJ, Li L (2015) Application study of comprehensive forecasting model based on entropy weighting method on trend of PM2.5 concentration in Guangzhou, China. Int J Environ Res Public Health 12(6):7085-99

31. Mahajan S, Liu HM, Tsai TC, Chen LJ (2018) Improving the accuracy and efficiency of $\mathrm{PM} 2.5$ forecast service using cluster-based hybrid neural network model. IEEE Access 6:19193-204

32. Wang P, Zhang H, Qin Z, Zhang G (2017) A novel hybrid-Garch model based on ARIMA and SVM for PM2.5 concentrations forecasting. Atmos Pollut Res 8(5):850-60

33. Feng X, Li Q, Zhu Y, Hou J, Jin L, Wang J (2015) Artificial neural networks forecasting of PM2.5 pollution using air mass trajectory based geographic model and wavelet transformation. Atmos Environ 107:118-28

34. Ausati S, Amanollahi J (2016) Assessing the accuracy of ANFIS, EEMD-GRNN, PCR, and MLR models in predicting PM2.5. Atmos Environ 142:465-74

35. Zhu D, Cai C, Yang T, Zhou X (2018) A machine learning approach for air quality prediction: model regularization and optimization. Big Data Cogn Comput 2(1):5

36. Kleine Deters J, Zalakeviciute R, Gonzalez M, Rybarczyk Y (2017) Modeling PM2.5 urban pollution using machine learning and selected meteorological parameters. J Electr Comput Eng. https ://doi.org/10.1155/2017/5106045
37. Soh PW, Chang JW, Huang JW (2018) Adaptive deep learningbased air quality prediction model using the most relevant spatial-temporal relations. IEEE Access 6:38186-99

38. Lin Y, Mago N, Gao Y, Li Y, Chiang YY, Shahabi C, Ambite JL (2018) Exploiting spatiotemporal patterns for accurate air quality forecasting using deep learning. In: Proceedings of the 26th ACM SIGSPATIAL international conference on advances in geographic information systems, pp 359-368

39. Li X, Peng L, Hu Y, Shao J, Chi T (2016) Deep learning architecture for air quality predictions. Environ Sci Pollut Res 23(22):22408-17

40. Sun W, Sun J (2017) Daily PM2.5 concentration prediction based on principal component analysis and LSSVM optimized by cuckoo search algorithm. J Environ Manag 188:144-52

41. Ni XY, Huang H, Du WP (2017) Relevance analysis and short-term prediction of PM2.5 concentrations in Beijing based on multisource data. Atmos Environ 150:146-61

42. EveryAware (2014) Final report on: sensor selection, calibration and testing; everyaware platform; smartphone applications. http://www.everyaware.eu/resources/deliverables/D1.2.pdf. Accessed 9 January 2019

43. Amaral S, de Carvalho J, Costa M, Pinheiro C (2015) An overview of particulate matter measurement instruments. Atmosphere 6(9):1327-45

44. Budde M, Zhang L, Beigl M (2014) Distributed, low-cost particulate matter sensing: scenarios, challenges, approaches. ProScience 1:230-6

45. Central Pollution Control Board (2011) Guidelines for the measurement of ambient air pollutants volume-II.http://www.india environmentportal.org.in/files/NAAQSManualVolumell.pdf. Accessed 2 December 2018

46. Environment S.A (2019) MP101M-Continuous, automatic PM10, PM2.5, PM1, TSP particulate monitor. http://www.envir onnement-sa.com/products-page/en/air-quality-monitoring -en/mp101m-continuous-automatic-pm10-pm2-5-pm1-tspparticulate-monitor/. Accessed 6 January 2019

47. Shah J, Mishra B (2020) loT-enabled low power environment monitoring system for prediction of PM2.5. Pervas Mobile Comput 67:101175

48. Baby A, Alexander AA (2018) A review on various techniques used in predicting pollutants. IOP Conf Ser Mater Sci Eng 396(1):012016

49. Mathbits (2018) Correlation cofficient. https://mathbits.com/ MathBits/TISection/Statistics2/correlation.htm. Accessed 27 December 2018

50. Icopal Noxite (2019) Nitrogen oxide (NOx) pollution. http:// www.m.icopal-noxite.co.uk/nox-problem/nox-pollution.aspx. Accessed 30 August 2019

51. World Health Organization (2019) Air pollution. https://www. who.int/airpollution/ambient/pollutants/en/. Accessed 31 August 2019

52. European Environment Agency (2019) Some common air pollutants. https://www.eea.europa.eu/publications/2599XXX/page0 08.html. Accessed 31 August 2019

53. Sharma B, Venugopalan K (2014) Comparison of neural network training functions for hematoma classification in brain $\mathrm{CT}$ images. IOSR J Comput Eng 16(1):31-5

54. The MathWorks Inc (2018) Train and apply multilayer neural networks. https://www.mathworks.com/help/deeplearning/ug/ train-and-apply-multilayer-neural-networks.html. Accessed 15 September 2018

55. The MathWorks Inc (2019) Multilayer shallow neural network architecture. https://in.mathworks.com/help/deeplearning/ 
ug/multilayer-neural-network-architecture.html. Accessed 30 August 2019

56. SGX sensortech (2019) The MICS-6814 is a compact MOS sensor with three fully independent sensing elements on one package.
https://sgx.cdistore.com/datasheets/sgx/1143_Datasheet $\% 20$ MiCS-6814\%20rev\%208.pdf. Accessed 2 January 2019

Publisher's Note Springer Nature remains neutral with regard to jurisdictional claims in published maps and institutional affiliations. 